\title{
Broadband reflective multi-polarization converter based on single- layer double-L-shaped metasurface
}

\author{
Chenyang Mao ${ }^{1} \cdot$ Yang Yang $^{1} \cdot$ Xiaoxiang He ${ }^{1}$. Jingming Zheng ${ }^{1} \cdot$ Chun Zhou $^{1}$
}

Received: 19 June 2017 / Accepted: 12 October 2017 / Published online: 13 November 2017

(C) The Author(s) 2017. This article is an open access publication

\begin{abstract}
In this paper, a broadband reflective multi-polarization converter based on single-layer double-L-shaped metasurface is proposed. The proposed metasurface can effectively convert linear-polarized (TE/TM) incident wave into the reflected wave with three different polarizations within the frequency bands of 5.5-22.75 GHz. Based on the electric and magnetic resonant features of the double-L-shaped structure, the proposed metasurface can convert linearly polarized waves into crosspolarized waves at three resonant frequency bands. Furthermore, the incident linearly polarized waves can be effectively converted into left/right handed circular-polarized (LHCP and RHCP) waves at other four non-resonance frequency bands. Thus, the proposed metasurface can be regarded as a seven-band multi-polarization converter. The prototype of the proposed polarization converter is analyzed and measured. Both simulated and measured results show the 3-dB axis ratio bandwidth of circular polarization bands and the high polarization conversion efficiency of cross-polarization bands when the incident wave changes from $0^{\circ}$ to $30^{\circ}$ at both TE and TM modes.
\end{abstract}

\section{Introduction}

Metasurface (MS), an artificial layer material with the thickness less than wavelength, which can realize flexible and effective regulation for electromagnetic wave phase, polarization mode or propagation mode, has received extensive attention in the past few years [1-3]. Polarization is one of the most important characteristics for the study of electromagnetic (EM) waves, which has widely utilized in many EM applications, such as in optical instrument, microwave communications and the manufacture of antennas [4-6]. Manipulation of polarization state is an inevitable link in many fields such as satellite communications, mobile communications and so on. Polarization converter is a kind of polarization control device which can effectively change the polarization state of incident wave. Conventional methods to manipulate polarizations include using refractive index gradient materials [7], anisotropic and chiral metamaterials [8-15]. However, the polarization converters based on these materials generally have narrow operating bandwidth. Due

Chenyang Mao

531357137@qq.com

1 The College of Electronic and Information Engineering, Nanjing University of Aeronautics and Astronautics

(NUAA), Nanjing, China to a lot of studies carried out on MS, broadband polarization converters have been achieved like a wideband cross-polarization converter using plasmon hybridizations in a ring/disk cavity [16] and the realization of a circular polarizationkeeping conversions based on a $\mathrm{N}$-shaped metasurface [17]. Whereas, for many applications such as remote sensors and radiometer [6], broadband multi-polarization converter is needed.

In this letter, a broadband multi-polarization converter based on single-layer double-L-shaped metasurface is proposed. For the linear-polarized (TE/TM) incident wave, the reflected wave is converted into left/right handed circular-polarized wave at the frequency bands of 5.5-6.1 and 12.1-16 GHz, while right-/left-handed circular-polarized wave at the frequency bands of $6.65-9.6$ and $18.1-22.5 \mathrm{GHz}$. Besides, for three bands of $6.15-6.45,10.2-11.45$ and $16.25-17.6 \mathrm{GHz}$, the reflected wave is converted into crosspolarized wave. Comparing with the previous designs [6, 18-22], the proposed single-layer double-L-shaped metasurface has lower profile, more operating frequency bands and wider bandwidth of polarization conversion. 


\section{Design of the polarization converter}

As given in [23], a microstructured surface is proposed for tuning the polarization state, phase difference of light in two orthogonal directions can be efficiently controlled through the metallic L-shaped structure. Based on this, a metallic double-L-shaped structure is presented to generate three resonances in this letter, which leads to a sevenband multi-polarization converter. As shown in the Fig. 1, the unit cell of MS structure is composed of two L-shaped metallic patches with different sizes and a square metallic ground. Geometrical parameters of the whole structure are chosen as follows: $p=6.4 \mathrm{~mm}, h=2.2 \mathrm{~mm}, l_{1}=2.4 \mathrm{~mm}$, $l_{2}=6 \mathrm{~mm}, w_{1}=0.3 \mathrm{~mm}, w_{2}=0.4 \mathrm{~mm}$. The substrate layer is FR4, with a dielectric constant of $\varepsilon_{\mathrm{r}}=4.4$ and a lost tangent of $\tan \delta=0.001$.

To study the polarization conversion performance of the proposed design, the numerical analysis of reflection amplitude and phase characteristics is carried out using Ansoft HFSS.

The proposed polarization converter is supposed to be placed on the $X O Y$ plane, due to the symmetry along the diagonal direction, only the $x$-polarized incidence is taken into consideration. Figure 2 shows the simulated reflection coefficient, $R_{x-x}$ is defined as the reflected coefficient of $x$-polarized reflected wave under the $x$-polarized normal incident wave, while $R_{y-x}$ is the reflected coefficient of y-polarized reflected wave. As shown in Fig. 2, the difference between magnitudes of $x$ - and $y$-polarized reflected wave is smaller than $-3 \mathrm{~dB}$ over the frequency bands 5.5-6.1, 6.65-9.6, 12.1-16 and 18.1-22.5 GHz, which indicates that $x$ - and $y$-polarized reflected waves have almost equal amplitude under the $x$-polarized incident wave in the

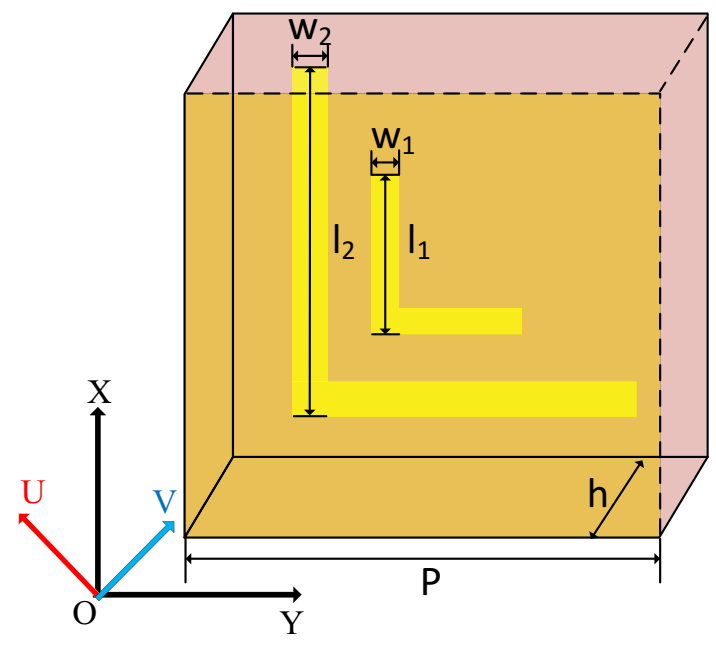

Fig. 1 The 3D view of MS unit cell

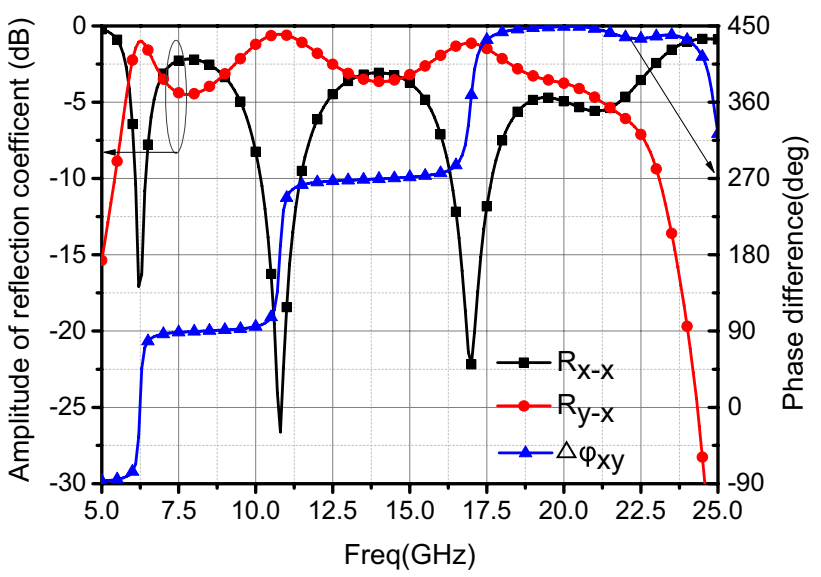

Fig. 2 Simulated amplitude of reflection coefficients under $x$-polarized incidence and phase difference of reflection coefficients under $x$-polarized incidence

four frequency bands. In addition, the phase difference of the reflection coefficients is given in Fig. 2, phase difference of $x$ - and $y$-polarized reflected wave is defined as $\Delta \varphi_{x y}$. As can be seen that the phase difference between $x$ - and $y$-polarized reflected waves approximately equals to $-90^{\circ}, 90^{\circ}, 270^{\circ}$, $450^{\circ}$ over the four frequency bands, respectively.

According to the simulated results shown in Fig. 2, it can be concluded that $x$-polarized normal incident wave is converted into left-handed circular-polarized reflected wave over 5.5-6.1 and 12.1-16 GHz, while right-handed circularpolarized reflected wave over $6.65-9.6$ and $18.1-22.5 \mathrm{GHz}$ using the proposed reflective metasurface. To make a further evaluation of the polarization conversion performance, the axis ratio (AR) of reflected wave is calculated by the formula [24] shown as follows:

$\mathrm{AR}=\left(\frac{\left|R_{x-x}\right|^{2}+\left|R_{y-x}\right|^{2}+\sqrt{a}}{\left|R_{x-x}\right|^{2}+\left|R_{y-x}\right|^{2}-\sqrt{a}}\right)^{\frac{1}{2}}$

$\begin{array}{lllllll}\mathrm{W} & \mathrm{h} & \mathrm{e} & \mathrm{r} & \mathrm{e} & \mathrm{i} & \mathrm{n}\end{array}$ $\mathrm{a}=\left|R_{x-x}\right|^{4}+\left|R_{y-x}\right|^{4}+2\left|R_{x-x}\right|^{2}\left|R_{y-x}\right|^{2} \cos \left(2 \Delta \varphi_{x y}\right)$. The calculated results are shown in Fig. 3, and it is shown that the axial ratio (AR) is lower than $3 \mathrm{~dB}$ in the frequency bands of 5.5-6.1, 6.65-9.6, 12.1-16 and 18.1-22.5 GHz. When the incident angle is changed from $0^{\circ}$ to $30^{\circ}$, the axial ratio characteristic almost keeps stable at low frequency bands, while at high frequency band changes violently.

In addition, based on the reflection coefficient $R_{x-x}$, it can be observed that there are three resonance frequencies (at $6.25,10.85$ and $16.95 \mathrm{GHz}$ ) in the frequency ranges of $6.15-6.45,10.2-11.45$ and $16.25-17.6 \mathrm{GHz}$, respectively. 


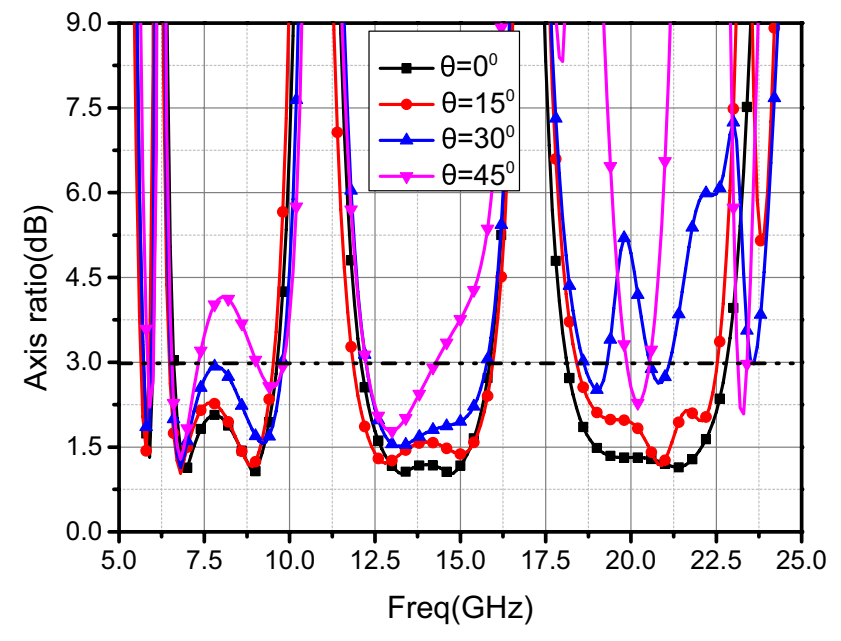

Fig. 3 The axis ratio (AR) of proposed polarization converter at the $x$-polarized incidence with different incident angles

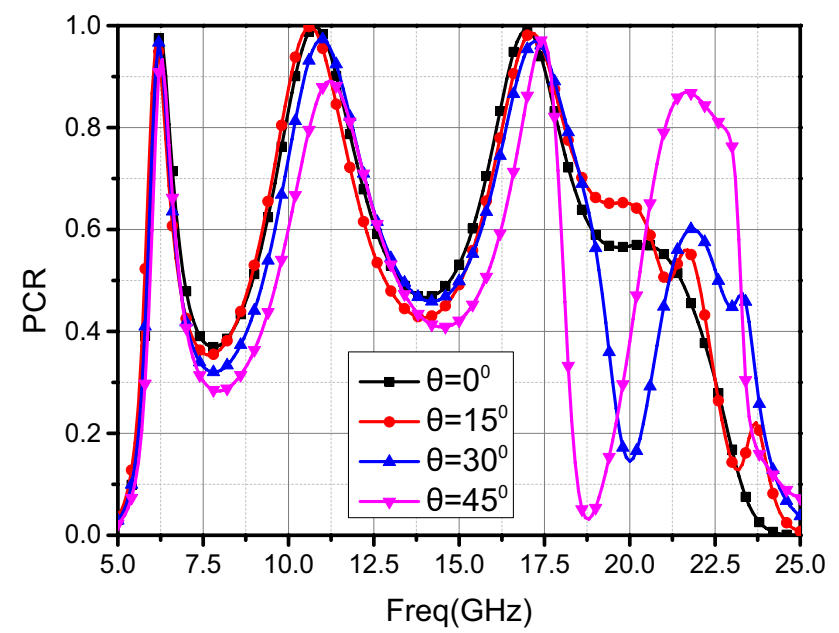

Fig. 4 The cross-polarization conversion rate (PCR) of proposed polarization converter at the $x$-polarized incidence with different incident angles

According to [25], the cross-polarization conversion rate (PCR) is defined as PCR $=R_{y-x}^{2} /\left(R_{x-x}^{2}+R_{y-x}^{2}\right)$, and it can be seen from Fig. 4 that the PCR is larger than $90 \%$ in the three frequency ranges given above, which indicates a good cross-polarization conversion performance. Considering the different incident angles, the three resonance frequencies are nearly unchanged when incident angle is changed from $0^{\circ}$ to $45^{\circ}$, meanwhile the PCR keeps above $90 \%$.

\section{Analysis of the proposed design}

To further understand the operating principle of the polarization rotation for the proposed metasurface, another analysis is presented using $u$ - and $v$-polarized normal incidence. As shown in Fig. 1, $u$ and $v$ are two mutually perpendicular symmetric axes along $\pm 45^{\circ}$ directions respected to $x$-direction. The surface current distributions of double-L-shaped metasurface and the metallic ground are simulated at resonant frequencies of $6.25,10.85$ and $16.95 \mathrm{GHz}$, respectively, as shown in Fig. 5a-c. At the resonant frequencies of 6.25 and $10.85 \mathrm{GHz}$, the most currents flow along the two arms of the large L-shaped resonator, while at the resonant frequency of $16.95 \mathrm{GHz}$ the most currents flow along the two arms of the small L-shaped resonator. Besides, the surface currents along the two L-shaped resonators at each resonant frequency produce induced currents on the metallic ground. As can be seen that, at frequency of $10.85 \mathrm{GHz}$, the most surface currents along the L-shaped resonators are parallel to the induced on the metallic ground, which generates the electric resonance. On the contrary, at frequencies of 6.25 and $16.95 \mathrm{GHz}$, the most surface currents along the L-shaped resonators are antiparallel to the induced currents, which generates magnetic resonances [26].

For $x$-polarized incidence, the incident wave is decomposed into two equal $u$ - and $v$-polarized components [24]:

$E_{i}=\left(u E_{u i}+v E_{v i}\right) e^{-j k z}$

And the reflected wave is given as:

$E_{r}=\left(u E_{u r} e^{j \varphi_{u}}+v E_{v r} e^{j \varphi_{v}}\right) e^{-j k z}$

As shown in Fig. 6a, it is indicated that there is a high co-polarization conversion rate under both $u$ - and $v$-polarized incidences, meanwhile the reflection coefficient $R_{u-u}$ approximately equals to $R_{v-v}$, which means that $E_{u r}$ will be approximately equal to $E_{v r}$. Considering the phase difference $\Delta \varphi_{u v}=\varphi_{u-u}-\varphi_{v-v}$, as shown in Fig. 6b, $\Delta \varphi_{u v}$ is close to $180^{\circ}$ in the frequency ranges of $6.15-6.45,10.2-11.45$ and $16.25-17.6 \mathrm{GHz}$, which leads to an orthogonal polarized reflected wave at the three bands.

In addition, $\Delta \varphi_{u v}$ is close to $90^{\circ}$ at the frequency bands of 5.5-6.1 and 12.1-16 GHz, and it is implied that two reflected components at the two equal $\mathrm{u}$ - and v-polarized incidences are combined into a right-handed circular-polarized wave in theses two frequency bands, while $\Delta \varphi_{u v}$ is close to $270^{\circ}$ at the frequency bands of $6.65-9.6$ and $18.1-22.5 \mathrm{GHz}$, so that the reflected wave will be left-handed circular-polarized under two equal $u$ - and $v$-polarized incidences.

\section{Measurement results}

For verification of the simulated results, a sample of the proposed metasurface is fabricated (shown in Fig. 7) on FR4 substrate by standard PCB etching and measured by an Agilent N5245A vector network analyzer in microwave anechoic chamber. A pair of transmitting and receiving horn. 
Fig. 5 Surface current distribution of the double-L-shaped metasurface and the metallic ground at the three resonance frequencies: a $f=6.25 \mathrm{GHz}$; b $f=10.85 \mathrm{GHz} ; \mathbf{c} f=16.95 \mathrm{GHz}$

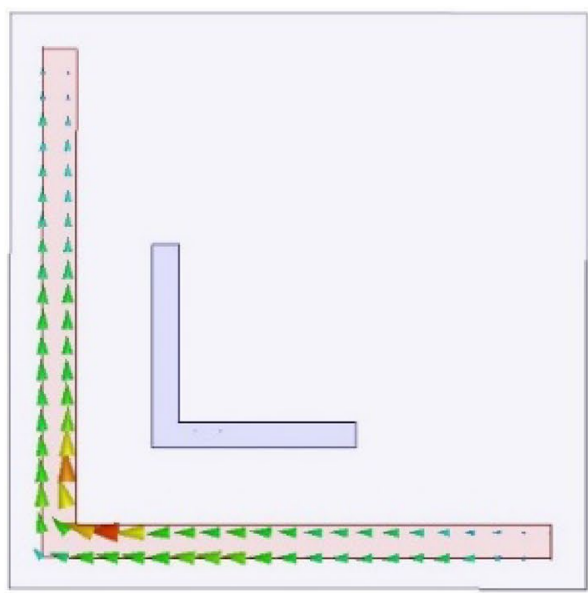

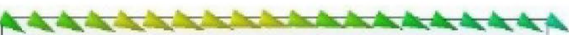

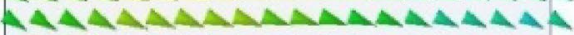

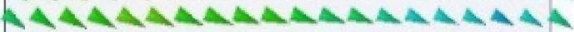

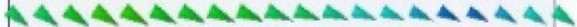

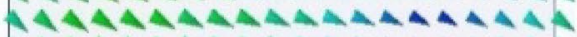

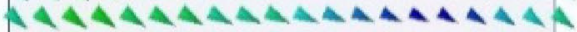

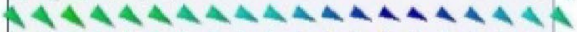

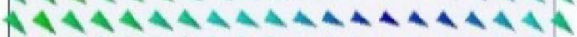

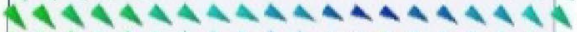

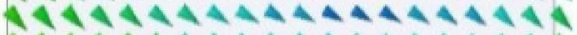

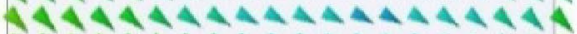

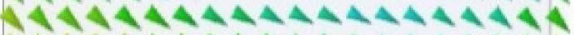

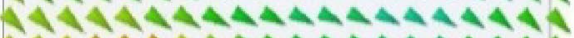

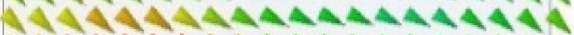

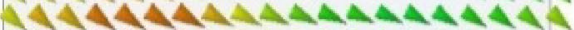

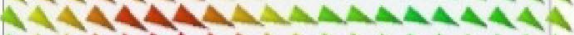

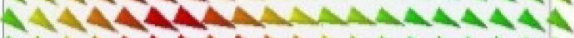

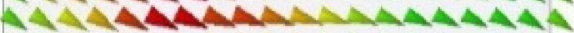

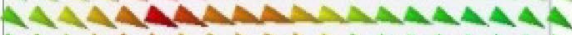

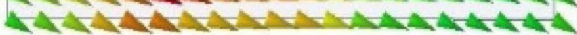

(a)
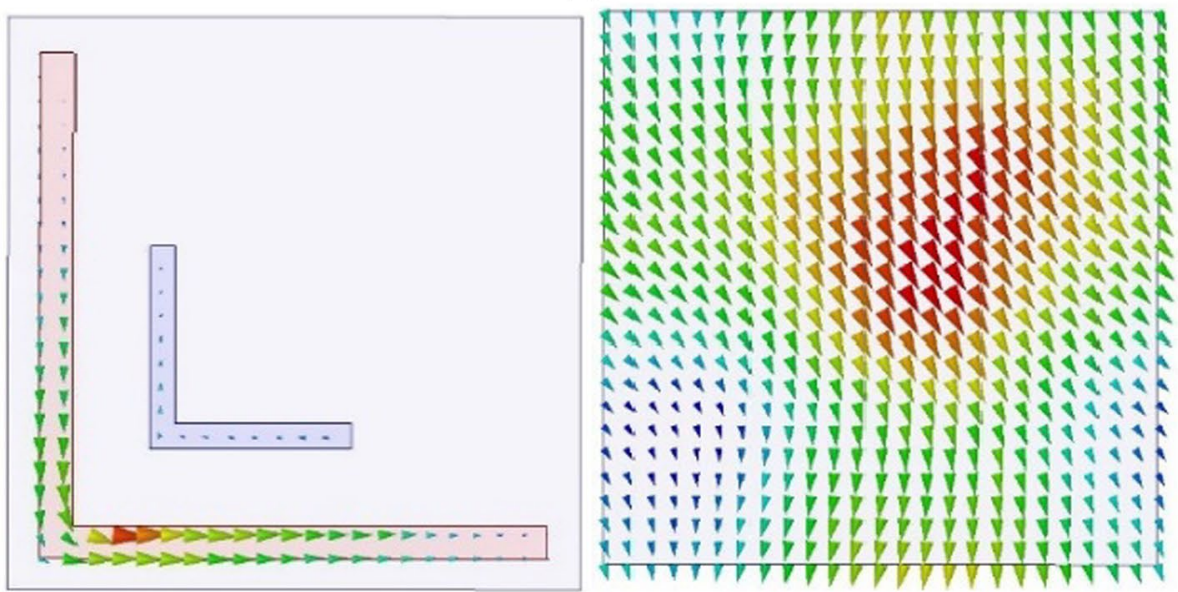

(b)
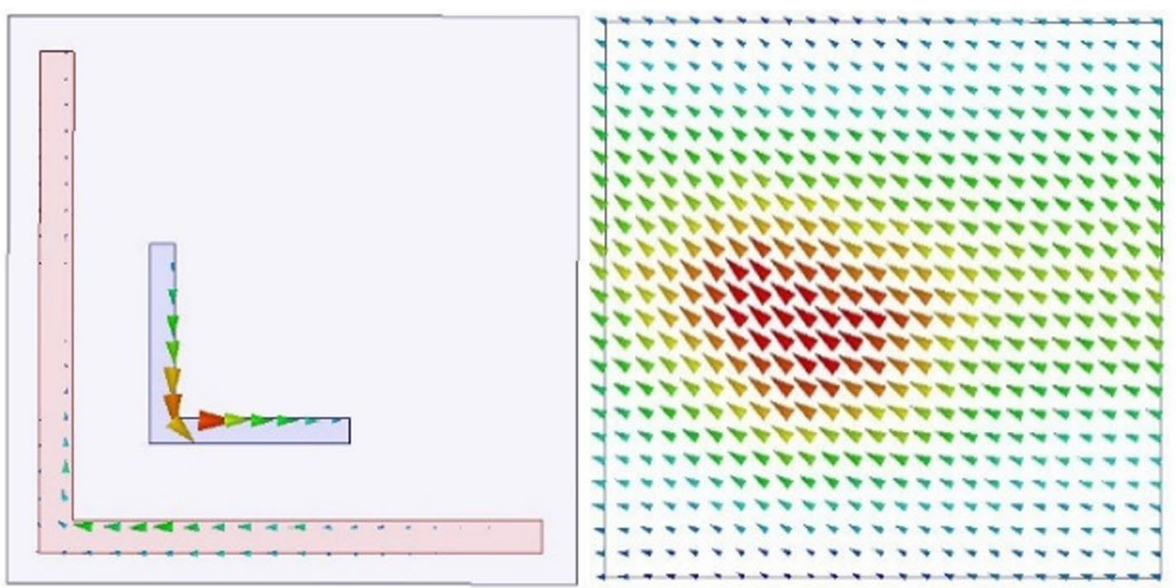

(c)

Antennas are connected to the two ports of a vector network analyzer, wherein the transmitting antenna is used to radiate $x$-polarized incident wave onto the metasurface, while the receiving one is used to receive $x$ - or $y$-polarized reflected waves. The comparison between simulated and measured results of axis ratio and cross-polarization conversion rate is given in Figs. 8 and 9, which shows a good agreement. 


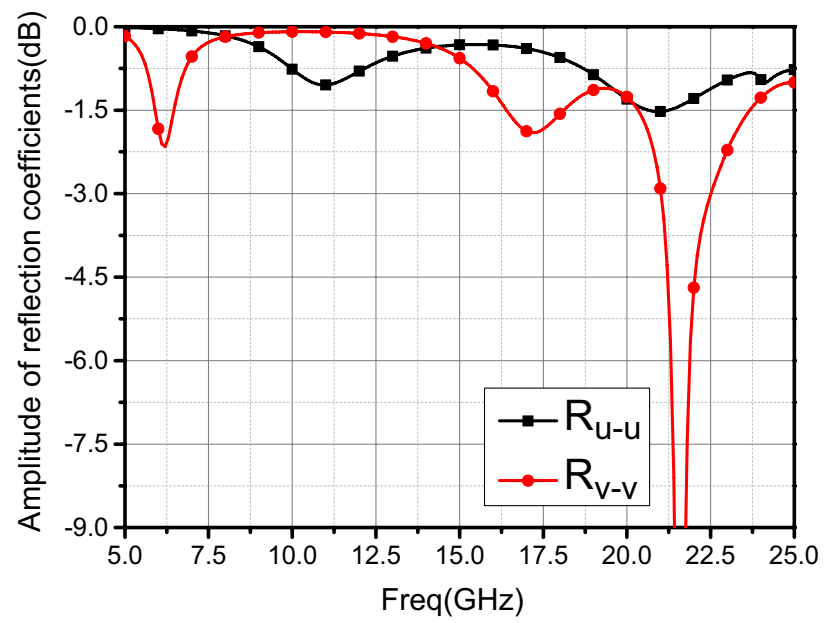

(a)

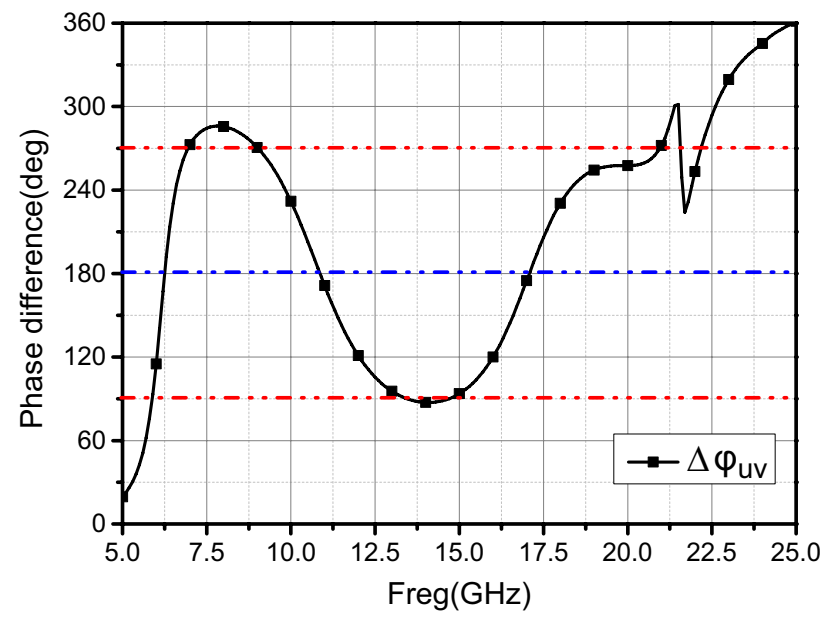

(b)

Fig. 6 a Simulated amplitude of reflection coefficients under $u$ - and $v$-polarized incidences, $\mathbf{b}$ simulated phase difference of reflection coefficients under $u$ - and $v$-polarized incidences

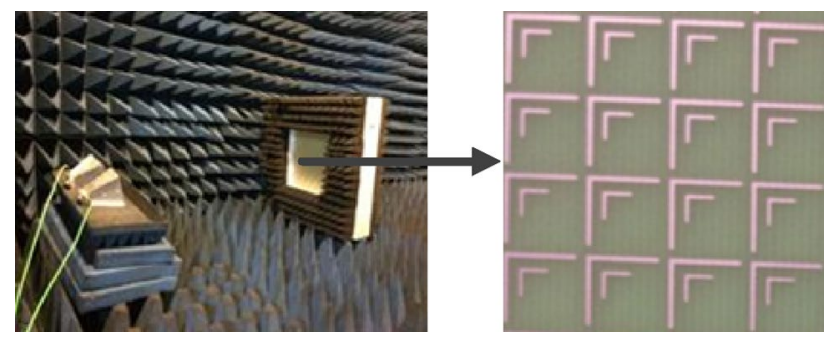

Fig. 7 Photograph of fabricated prototype

\section{Conclusion}

In conclusion, a broadband reflective multi-polarization converter based on single-layer double-L-shaped

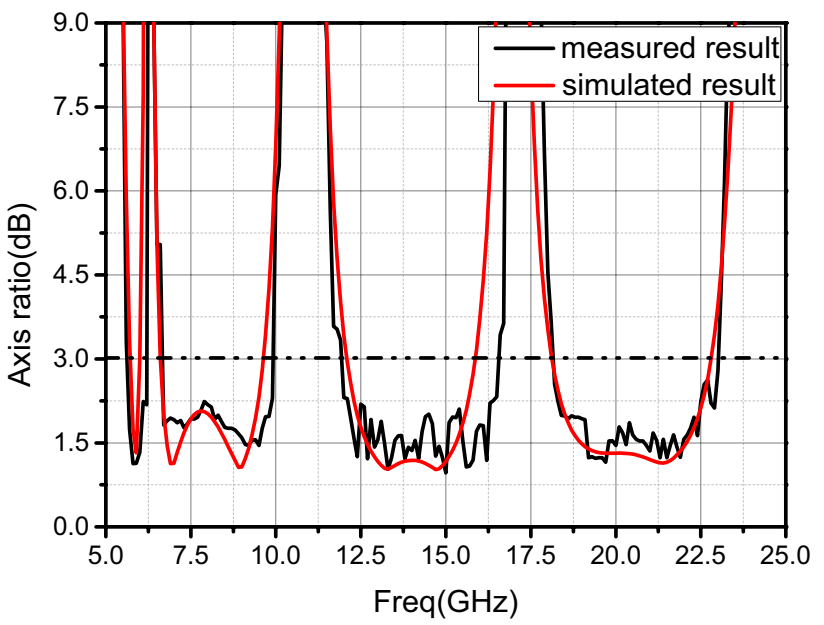

Fig. 8 The simulated and measured axis ratio (AR) of proposed polarization converter at the $x$-polarized normal incident wave

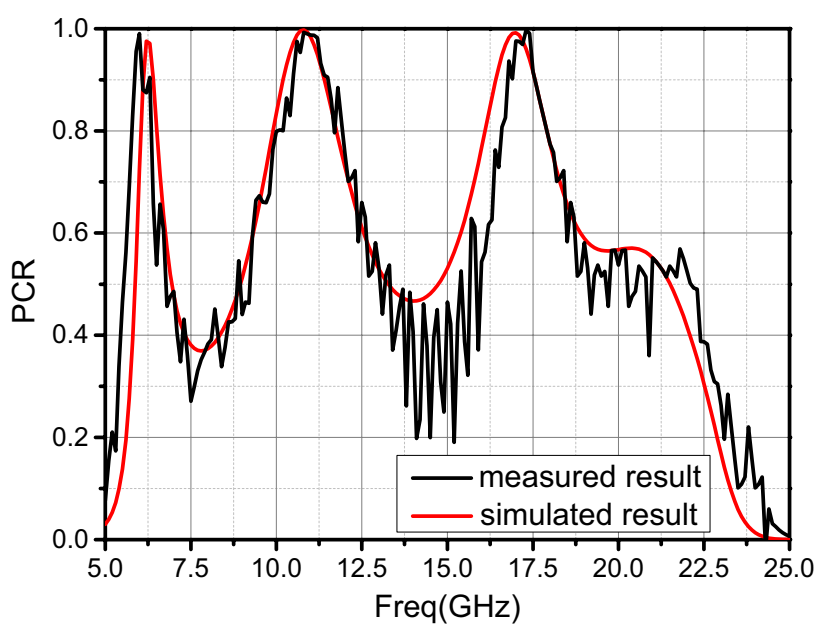

Fig. 9 The simulated and measured cross-polarization conversion rate (PCR) of proposed polarization converter at the x-polarized normal incident wave

metasurface is designed. The metasurface structure can be regarded as a seven-band polarization converter, for the linear-polarized (TE/TM) incident wave, at the frequency bands of 5.5-6.1 and 12.1-16 GHz, the reflected wave is converted into left-/right-handed circular-polarized wave, while right-/left-handed circular-polarized wave at the frequency bands of $6.65-9.6$ and $18.1-22.5 \mathrm{GHz}$, and for the three bands of $6.15-6.45,10.2-11.45$ and $16.25-17.6 \mathrm{GHz}$, the reflected wave is converted into a cross-polarized wave. Besides, it keeps good conversion performance when the incident wave changes from $0^{\circ}$ to $30^{\circ}$ at both TE and TM modes. The working principle of the proposed polarization conversion is analyzed and explained in the paper. 
Acknowledgements This work was supported by the Foundation of Graduate Innovation Center in NUAA (Grant No. kfjj20160411).

Open Access This article is distributed under the terms of the Creative Commons Attribution 4.0 International License (http://creativecommons.org/licenses/by/4.0/), which permits unrestricted use, distribution, and reproduction in any medium, provided you give appropriate credit to the original author(s) and the source, provide a link to the Creative Commons license, and indicate if changes were made.

\section{References}

1. C.L. Holloway et al., Metamaterials 3, 100-112 (2009)

2. C.L. Holloway et al., IEEE Antennas Propag. Mag 54, 10-35 (2012)

3. C.L. Holloway, D. Love, E.F. Kuester, J.A. Gordon, D.A. Hill, IEEE Trans. Antennas Propag. 60, 5173-5186 (2012)

4. C. Dietlein, A. Luukanen, Z.B. Popovic, E. Grossman, A W-band polarization converter and isolator. IEEE Trans. Antennas Propag. 55, 1804-1809 (2007)

5. M. Euler, V. Fusco, R. Cahill, R. Dickie, IEEE Trans. Antennas Propag. 58, 2457-2459 (2010)

6. J.X. Zhao, B.X. Xiao, X.J. Huang et al., Microw. Opt. Technol. Lett. 57, 978-983 (2015)

7. X.B. Liu, H.Y. Shi, B. Chen, Y.S. Jiang, Z. Xu, A.X. Zhang, Acta Phys. Sin 63, 214201-214201 (2014)
8. L. Cong, W. Cao, Z. Tian et al., New J. Phys 14, 115013 (2012)

9. X. Ma, C. Huang, M. Pu et al., Opt. Express 20, 16050 (2012)

10. Y. Zhao, A. Alù, Phys. Rev. B 84, 205428 (2011)

11. J. Shi, X. Liu, S. Yu, Appl. Phys. Lett. 102, 917 (2013)

12. J. Han, H. Li, Y. Fan, Appl. Phys. Lett. 98, 1996 (2011)

13. A.E. Akosman, A.E. Serebryannikov, E. Ozbay, Opt. Lett. 36, $1653(2011)$

14. K. Song, X. Zhao, Y. Liu, Appl. Phys. Lett. 103, 1353 (2013)

15. Y. Ye, S. He, Appl. Phys. Lett. 96, 788 (2010)

16. H. Shi, J. Li, A. Zhang, J. Wang, Z. Xu, Opt. Express 22, 2097320981 (2014)

17. Y. Li, J. Zhang, S. Qu, J. Wang, L. Zheng, Z. Zhou, Z. Xu, A. Zhang, Chin. Phys. B 24, 014202 (2015)

18. X. Huang, D. Yang, H. Yang, J. Appl. Phys. 115, 2494 (2014)

19. X. Huang, B. Xiao, L. Guo, J. Opt. 16, (2014)

20. H.F. Ma, G.Z. Wang, G.S. Kong, Opt. Mater. Exp. 4, 1717-1724 (2014)

21. H. Li, B. Xiao, X. Huang, Phys. Script., 90 (2015)

22. X. Liu, J. Zhang, W. Li, IEEE Antennas Wirel. Propag. Lett 99, $1-1(2016)$

23. S. Chi, X. Xiong, Y. Hu, G. Ma, R. Peng, C. Sun, M. Wang, Phys. Rev. X 4, 021026 (2014)

24. B. Lin, J.L. Wu, X.Y. Da, Appl. Phys. A 123, 43 (2017)

25. S.A. Winkler, W. Hong, M. Bozzi, K. Wu, IEEE Trans. Antennas Propag 58, 1202-1213 (2010)

26. X. Gao, X. Han, W.P. Cao, IEEE Trans. Antennas Propag 63 , 3522-3530 (2015) 